\title{
On Quasiminimal Excellent Classes
}

\author{
Jonathan Kirby*
}

August 14, 2012

\begin{abstract}
A careful exposition of Zilber's quasiminimal excellent classes and their categoricity is given, leading to two new results: the $L_{\omega_{1}, \omega}(Q)$ definability assumption may be dropped, and each class is determined by its model of dimension $\aleph_{0}$.
\end{abstract}

Boris Zilber developed quasiminimal excellent classes in [Zil05] in order to prove that his conjectural description of complex exponentiation was uncountably categorical, that is, it has exactly one model of each uncountable cardinality. This article gives a simplified and careful exposition of quasiminimal excellent classes, and of the categoricity proof. This more careful exposition has led to two new results. We say that a quasiminimal excellent class is degenerate iff either it has only finite dimensional models, or it is a proper subclass of another quasiminimal excellent class. These are essentially uninteresting cases (at least in the context for which quasiminimal excellent classes were invented). In [Zil05], the proof of the existence of arbitrarily large models depended on the class being definable by an $L_{\omega_{1}, \omega}(Q)$-sentence of a specific form (and on having an infinite-dimensional model). $Q$ is the quantifier "there exist uncountably many". The question of whether this could be generalized to any $L_{\omega_{1}, \omega}(Q)$-sentence was posed. Here we show that every nondegenerate quasiminimal excellent class is definable by an $L_{\omega_{1}, \omega}(Q)$ sentence of the specific form given, hence is uncountably categorical. Furthermore, any quasiminimal excellent class with an infinite-dimensional model extends uniquely to a nondegenerate class, and hence a quasiminimal excellent class may be produced in a "bootstrap" fashion from its unique model of dimension $\aleph_{0}$.

\footnotetext{
${ }^{*}$ University of Oxford and University of Illinois at Chicago, supported by the EPSRC fellowship EP/D065747/1
} 
This article sprang from many lively and productive discussions I had with John Baldwin. The account of quasiminimal excellent classes in Bal07] was rewritten in conjunction with this article, and incorporates many of the ideas given here. Some of the differences between this account of the categoricity proof and Boris Zilber's are due to John Baldwin, in particular the construction of the isomorphism in theorem 3.3 as the union of the maps $f_{X}$, and the introduction of the Shelah-style statement of excellence in lemma 3.2 .

\section{The Definition}

The languages $L$ we consider are the same as those considered in first-order model theory, with relation symbols and function symbols (each of finite arity) and constant symbols. We assume for convenience that the languages are one-sorted, although in fact multi-sorted languages would not give any greater generality in the context of this paper.

Given $L$-structures $H$ and $H^{\prime}$, a partial embedding $f: H \rightarrow H^{\prime}$ consists of $L$-substructures $X \subseteq H$ and $X^{\prime} \subseteq H^{\prime}$ and an $L$-isomorphism $X \cong X^{\prime}$, also called $f . X$ is called the preimage and $X^{\prime}$ is called the image of $f$. If $X, X^{\prime}$ are subsets of $H, H^{\prime}$ respectively, and $f: X \rightarrow X^{\prime}$ is a bijective function, then there is at most one way to extend $f$ to an $L$-isomorphism between the substructures of $H$ and $H^{\prime}$ generated by $X$ and $X^{\prime}$. If this extension of $f$ exists we also say that $f$ is a partial embedding. Note that there is a partial embedding with preimage $X$ and image $X^{\prime}$ precisely when $X$ and $X^{\prime}$ have the same quantifier-free $L$-type.

The central notion in the definition of a quasiminimal excellent class is that of a pregeometry, and we refer the reader unfamiliar with this concept to [Mar02, p289] or to most other books on model theory or stability theory.

Definition 1.1. A quasiminimal excellent class is a class $\mathcal{C}$ of pairs $\left\langle H, \mathrm{cl}_{H}\right\rangle$ such that $H$ is an $L$-structure (for a given language $L$ ) and $\mathrm{cl}_{H}$ is a function $\mathcal{P} H \stackrel{\mathrm{cl}_{H}}{\longrightarrow} \mathcal{P} H$ (where $\mathcal{P} H$ is the power set of $H$ ), satisfying axioms 0 , I, II, and III.

\section{0: Closure under isomorphism}

Suppose that $\left\langle H, \mathrm{cl}_{H}\right\rangle$ and $\left\langle H^{\prime}, \mathrm{cl}_{H^{\prime}}\right\rangle$ are $L$-structures equipped with functions on their powersets, that $\left\langle H, \mathrm{cl}_{H}\right\rangle \in \mathcal{C}$, and that there is an $L$-isomorphism $f: H \cong H^{\prime}$ such that for every $y \in H$ and $X \subseteq H$ we have $y \in \operatorname{cl}_{H}(X)$ iff $f(y) \in \operatorname{cl}_{H^{\prime}}(f(X))$. Then $\left\langle H^{\prime}, \operatorname{cl}_{H^{\prime}}\right\rangle \in \mathcal{C}$.

\section{I: Pregeometry and its relationship to $L$}


I.1 For each $\left\langle H, \mathrm{cl}_{H}\right\rangle \in \mathcal{C}, \mathrm{cl}_{H}$ is a pregeometry on $H$, satisfying the countable closure property $(\mathrm{CCP})$ : the closure of any finite set is countable.

I.2 If $\left\langle H, \mathrm{cl}_{H}\right\rangle \in \mathcal{C}$ and $X \subseteq H$, then $\operatorname{cl}_{H}(X)$ equipped with the restriction of $\mathrm{cl}_{H}$ is in $\mathcal{C}$.

I.3 If $\left\langle H, \mathrm{cl}_{H}\right\rangle,\left\langle H^{\prime}, \mathrm{cl}_{H^{\prime}}\right\rangle \in \mathcal{C}, X \subseteq H, y \in \mathrm{cl}_{H}(X)$, and $f: H \rightarrow H^{\prime}$ is a partial embedding with $X \cup\{y\} \subseteq \operatorname{preim}(f)$, then $f(y) \in$ $\operatorname{cl}_{H^{\prime}}(f(X))$.

\section{II: $\aleph_{0}$-homogeneity over countable models}

Let $\left\langle H, \mathrm{cl}_{H}\right\rangle,\left\langle H^{\prime}, \mathrm{cl}_{H^{\prime}}\right\rangle \in \mathcal{C}$, let $G \subseteq H$ and $G^{\prime} \subseteq H^{\prime}$ be countable closed subsets or the empty set, and let $g: G \rightarrow G^{\prime}$ be an isomorphism.

II.1 If $x \in H$ and $x^{\prime} \in H^{\prime}$, independent from $G$ and $G^{\prime}$ respectively, then $g \cup\left\{\left(x, x^{\prime}\right)\right\}$ is a partial embedding.

II.2 If $g \cup f: H \rightarrow H^{\prime}$ is a partial embedding, $f$ has finite preimage $X$, and $y \in \operatorname{cl}_{H}(X \cup G)$, then there is $y^{\prime} \in H^{\prime}$ such that $g \cup f \cup\left\{\left(y, y^{\prime}\right)\right\}$ is a partial embedding.

A crown in $H$ is a subset $C \subseteq H$ such that there is an independent subset $B$ of $H$ (independent with respect to the pregeometry $\mathrm{cl}_{H}$ ) and finitely many subsets $B_{1}, \ldots, B_{n}$ of $B$ such that $C=\bigcup_{i=1}^{n} \mathrm{cl}_{H} B_{i}$. A partial embedding $f: H \rightarrow H^{\prime}$ is said to be closed iff for every closed set $X$ in the preimage of $f$, the image $f(X)$ is closed in $H^{\prime}$.

\section{III: Quasiminimal excellence}

Let $H, H^{\prime} \in \mathcal{C}$, let $C$ be a countable crown in $H$, and let $g: H \rightarrow H^{\prime}$ be a closed partial embedding with preimage $C$. For any finite subset $X$ of $\mathrm{cl}_{H}(C)$, there is a finite subset $C_{0}$ of $C$ such that if $f: H \rightarrow H^{\prime}$ has preimage $X$ and $\left.f \cup g\right|_{C_{0}}$ is a partial embedding then $f \cup g$ is also a partial embedding. We say that the quantifier-free type of $X$ over $C$ is determined over $C_{0}$.

The reader may like to have some examples in mind.

Examples 1.2. The following are quasiminimal excellent classes.

1. Any strongly minimal theory in a countable language such that the algebraic closure of the empty set is infinite, with algebraic closure. (These are elementary classes.) 
2. The class of all $L$-structures where $L$ is the empty language (so $L$ structures are just sets), with $\operatorname{cl}_{H}(X)=X$. (This is an elementary class but not a complete theory, since to satisfy axiom I.2 we include the finite models.)

3. $\mathrm{ACF}_{0}$, with a predicate $Z$ and the axiom $Z(x) \longleftrightarrow \bigvee_{n \in \mathbb{Z}} x=n$, with algebraic closure (an $L_{\omega_{1}, \omega}$-class).

4. The theory consisting just of one equivalence relation, all of whose blocks have size $\aleph_{0}$, the closure of a subset $X$ being the union of the blocks meeting $X$ (an $L(Q)$-class).

5. Take $L$ to be the language with just two unary relation symbols, $B$ and $R$, and consider the class $\mathcal{C}_{B}$ of $L$-structures in which every element lies in exactly one of the sets $B$ and $R$, and $R$ has size $\aleph_{0}$. Define $\mathrm{cl}_{H}^{B}$ on $H \in \mathcal{C}_{B}$ by $\operatorname{cl}_{H}^{B}(X)=(X \cap B) \cup R$.

6. The motivating example: Zilber's exponential field, and the related examples of "covers of the multiplicative group" and "raising to powers".

Axiom I.3 says that the pregeometry is determined entirely by the $L$ structure. Indeed, if $f: H \rightarrow H^{\prime}$ is a partial embedding then so is its inverse, $f^{-1}: H^{\prime} \rightarrow H$, so I.3 can be strengthened to $y \in \operatorname{cl}_{H}(X)$ iff $f(y) \in$ $\mathrm{cl}_{H^{\prime}}(f(X))$. In particular, if $X$ and $X^{\prime}$ have the same quantifier-free $L$ type, then the restriction of the pregeometries to them is the same. Since the pregeometry is determined by the language, from now on we write just $H \in \mathcal{C}$ rather than $\left\langle H, \mathrm{cl}_{H}\right\rangle \in \mathcal{C}$. Note however that in specifying the class $\mathcal{C}$ we do have to specify the pregeometry, because there may be more than one option. For example, in example 1.2 .5 above, we could also define a pregeometry $\mathrm{cl}_{H}^{R}(X)=(X \cap R) \cup B$ on each $H \in \mathcal{C}$. Then both pregeometries satisfy axiom I.3, although to form a quasiminimal excellent class with $\mathrm{cl}^{R}$ we would need to choose a different class of $L$-structures where $B$ was fixed and $R$ varied.

For any $H \in \mathcal{C}$, the pregeometry $\mathrm{cl}_{H}$ gives a notion of an independent subset of $H$, and the dimension of $H$ is defined to be the (well-defined) cardinality of a maximal independent subset of $H$. Similarly, the dimension of a subset $X \subseteq H$ is defined to be the cardinality of a maximal independent subset of $X$. The restriction of $\mathrm{cl}_{H}$ to $X$ depends only on the quantifier-free type of $X$, as above, so it follows that the dimension of $X$ only depends on its quantifier-free type, and not on how it is embedded into $H$.

Definition 1.3. We consider a class $\mathcal{C}$ satisfying axioms 0 and I as a category by taking the closed embeddings, that is, those $L$-embeddings $H \hookrightarrow H^{\prime}$ such 
that the image of $H$ is closed in $H^{\prime}$. Write $H \preccurlyeq H^{\prime}$ if the inclusion $H \subseteq H^{\prime}$ is a closed embedding.

Example 1.2, 4 above is the simplest in which not all submodels are closed submodels. The notion of a closed $L$-embedding also preserves the closure operator.

Lemma 1.4. If $H, H^{\prime} \in \mathcal{C}$ with $H \subseteq H^{\prime}$ and $X$ is any subset of $H$ then $\mathrm{cl}_{H}(X)=\mathrm{cl}_{H^{\prime}}(X) \cap H$. Furthermore, if $H \preccurlyeq H^{\prime}$ then $\mathrm{cl}_{H}(X)=\mathrm{cl}_{H^{\prime}}(X)$. In particular, a closed embedding is a closed partial embedding (with respect to the definitions above).

Proof. The first statement is axiom I.3 applied to the inclusion map $H \hookrightarrow H^{\prime}$. For the second statement, since $X \subseteq H$ we have $\mathrm{cl}_{H^{\prime}}(X) \subseteq \mathrm{cl}_{H^{\prime}}(H)=H$, and so $\mathrm{cl}_{H^{\prime}}(X) \cap H=\mathrm{cl}_{H^{\prime}}(X)$.

Axioms II and III are stated in rather technical terms, and are best understood by following through the subsequent proofs where they are used. A crown is a finite union of closed sets in a particular configuration, sometimes known as an independent $n$-system or independent n-cube. The excellence axiom amounts to the existence and uniqueness of amalgams of crowns. I have introduced the terminology crown which is shorter than the alternatives, does not contain the awkward parameter $n$, and which I think is a better description of the concept. Zilber has also used special subset. The terminology of a type being determined over a set is preferred to Zilber's original defined over a set, which conflicts with a different usage in stability theory.

The definition of a quasiminimal excellent class given here differs from Zilber's in the following ways. The pregeometry is included as data rather than its existence being postulated as an axiom. This avoids ambiguity where there may be more than one pregeometry to choose from, as in example 1.2 .5 above. The exchange and CCP axioms for the pregeometry are included in the definition. Early versions of Zilber's axiomatization omitted exchange, but he later realized it was necessary. In the original definition, only the models with CCP were of interest (indeed only they were quasiminimal), but for technical reasons CCP was not included as an axiom. The technical reasons are avoided by considering a quasiminimal excellent class as a category with closed embeddings (which are also introduced here). Axiom I.3 was listed among the homogeneity axioms rather than the pregeometry axioms in Zil05, and is here made precise. Axiom II is now $\aleph_{0}$-homogeneity over countable, closed submodels, not all submodels. II.1 is weakened to consider only singletons, not independent tuples of arbitrary length. The excellence axiom III is stated only for countable crowns. These weakenings of 
the axioms to countable models and the corresponding strengthening of the categoricity theorem are the main reason why the new results of this paper can be proved.

The axioms all refer to partial embeddings, and imply that the language $L$ is rich enough to have a form of quantifier elimination (see proposition 3.5). This is a minor convenience, but is not necessary. In examples, this quantifier elimination must usually be obtained by first expanding the language. It would perhaps be better to define a quasiminimal excellent class to be any class $\mathcal{C}^{\prime}$ of $L^{\prime}$-structures for which there is an expansion by definitions to a language $L$, such that the resulting class $\mathcal{C}$ satisfies the given axioms.

In section 4, I introduce additional axioms IV on unions of chains and the existence of an infinite-dimensional model. The subsequent analysis shows that adding these axioms rules out exactly the degenerate cases, hence it would be natural and would do no harm to add them to the definition of a quasiminimal excellent class, but that convention is not adopted in this paper.

\section{Uniqueness of models up to dimension $\aleph_{1}$}

We first show that the main theorem about quasiminimal excellent classes holds for models up to size $\aleph_{1}$, without assuming the excellence axiom. Thus if one's interest is only in structures of size $2^{\aleph_{0}}$ such as expansions of the complex field, and one is prepared to assume the continuum hypothesis, then one can prove categoricity results without reference to excellence.

Theorem 2.1. Let $\mathcal{C}$ be a class satisfying axioms 0 , I and II, and let $H, H^{\prime} \in$ $\mathcal{C}$ with $\operatorname{dim} H \leqslant \aleph_{1}$. Let $G \subseteq H$ be empty or closed and countable, and let $f_{0}: G \rightarrow H^{\prime}$ be a closed embedding (or the empty map if $G$ is empty). Let $B$ be a basis of $H$ over $G$ and suppose $\psi_{B}: H \rightarrow H^{\prime}$ is an injective partial map with preimage $B$ and image an independent set over $\operatorname{Im} f_{0}$. Then $\psi:=f_{0} \cup \psi_{B}$ extends to a closed map $\hat{\psi}: H \rightarrow H^{\prime}$.

In particular, if $\operatorname{Im} \psi$ spans $H^{\prime}$ then $\hat{\psi}$ is an isomorphism.

Proof. Well-order $B$ as $\left(b_{\lambda}\right)_{\lambda<\mu}$ for some ordinal $\mu \leqslant \omega_{1}$. For each ordinal $\nu \leqslant \mu$, let $G_{\nu}=\operatorname{cl}_{H}\left(G \cup\left\{b_{\lambda} \mid \lambda<\nu\right\}\right)$. In particular, $G_{0}=G$. Inductively, we construct closed maps $f_{\nu}: G_{\nu} \rightarrow H^{\prime}$ such that

- $\psi \uparrow_{G_{\nu}} \subseteq f_{\nu}$, and

- If $\nu_{1} \leqslant \nu_{2}$ then $f_{\nu_{1}} \subseteq f_{\nu_{2}}$.

At limit ordinals, take unions. For a successor $\nu=\lambda+1$, we construct $f_{\nu}$ as $\bigcup_{n \in \mathbb{N}} h_{n}$ where the $h_{n}$ are partial embeddings constructed inductively by the back and forth method, such that $h_{0}=\left\{\left(b_{\lambda}, \psi\left(b_{\lambda}\right)\right)\right\}$, and for each $n$, 
- $\operatorname{preim}\left(h_{n}\right)$ is finite,

- $h_{n} \subseteq h_{n+1}$, and

- $h_{n} \cup f_{\lambda}$ is a partial embedding.

Both $G_{\nu}$ and $G_{\nu}^{\prime}:=\operatorname{cl}_{H^{\prime}}\left(f_{\lambda}\left(G_{\lambda}\right) \cup \psi\left(b_{\lambda}\right)\right)$ are countable, so list their elements in chains of length $\omega$.

By axiom II.1, $h_{0} \cup f_{\lambda}$ is a partial embedding. For odd $n$, let $a$ be the least element of $G_{\nu} \backslash$ preim $h_{n-1}$. By axiom II.2, there is $b \in G_{\nu}^{\prime}$ such that, taking $h_{n}:=h_{n-1} \cup\{(a, b)\}, h_{n} \cup f_{\lambda}$ is a partial embedding.

For even $n \geqslant 2$, repeat the process going back rather than forth. Then $f_{\nu}$ is a embedding defined on all of $G_{\nu}$, with image all of $G_{\nu}^{\prime}$, hence it is a closed embedding. It extends $f_{\lambda}$ and $\psi \uparrow_{G_{\nu}}$ by construction.

Take $\hat{\psi}=\bigcup_{\lambda<\mu} f_{\lambda}$. This is a closed embedding as required.

Corollary 2.2 (Uniqueness of models up to $\aleph_{1}$ ). Models of a quasiminimal excellent class of dimension less than or equal to $\aleph_{1}$ are determined up to isomorphism by their dimension. There is at most one model of cardinality $\aleph_{1}$.

A small modification of the proof explains why axiom II is called $\aleph_{0^{-}}$ homogeneity over countable models.

Proposition 2.3. Let $\mathcal{C}$ be a class satisfying axioms $0, I$ and $I I$, let $H \in \mathcal{C}$, let $G \subseteq H$ be empty or countable and closed, and let $\bar{x}, \bar{y}$ be $n$-tuples from $H$ with the same quantifier-free type over $G$. Then there is an automorphism $\sigma \in \operatorname{Aut}(H / G)$ such that $\sigma(\bar{x})=\bar{y}$.

Proof. Write the tuple $\bar{x}$ as $b_{1}, \ldots, b_{n}, a_{1}, \ldots, a_{m}$ where the $b_{i}$ are independent over $G$ and each $a_{i}$ lies in $\mathrm{cl}_{H}\left(G b_{1}, \ldots, b_{n}\right)$, and correspondingly write $\bar{y}$ as $b_{1}^{\prime}, \ldots, b_{n}^{\prime}, a_{1}^{\prime}, \ldots, a_{m}^{\prime}$. By axiom I.3 and the discussion after examples 1.2 , the pregeometry is determined by the quantifier-free $L$-type, and hence $b_{1}^{\prime}, \ldots, b_{n}^{\prime}$ are independent over $G$, and each $a_{i}^{\prime}$ lies in $\mathrm{cl}_{H}\left(G b_{1}^{\prime}, \ldots, b_{n}^{\prime}\right)$. Extend $\bar{b}$ and $\bar{b}^{\prime}$ to bases of $H$ over $G$, and let $\psi_{B}$ be a bijection between the bases which sends $b_{i}$ to $b_{i}^{\prime}$ for $i=1, \ldots, n$. Let $f_{0}$ be the identity map on $G$. Then follow the proof of theorem 2.1, except that in the construction of $f_{\nu}$ for $\nu \leqslant n$, start by sending $a_{i}$ to $a_{i}^{\prime}$ for each $i$ such that $a_{i} \in \operatorname{cl}_{H}\left(G b_{1}, \ldots, b_{\nu}\right)$.

Note that the construction in the proof of theorem 2.1 cannot be carried out for $H$ of dimension greater than $\aleph_{1}$, because we only have $\aleph_{0}$-homogeneity over models, not $\aleph_{1}$-homogeneity over models. (In addition, we have only assumed homogeneity over countable models, but we show later that is not an issue, whereas most examples are actually not $\aleph_{1}$-homogeneous over models.) 


\section{Uniqueness of large models}

Lemma 3.1. Let $M$ be a set, let $\mathrm{cl}$ be a pregeometry on $M$ and let $B$ be an independent subset of $M$. Let $X, Y \subseteq B$. Then $\operatorname{cl}(X) \cap \operatorname{cl}(Y)=\operatorname{cl}(X \cap Y)$.

Proof. It is convenient to use a notational shorthand. For subsets $X, Y$, and elements $x, y$, we write $\operatorname{cl}(X Y)$ to mean $\operatorname{cl}(X \cup Y) \operatorname{cl}(X x)$ to mean $\operatorname{cl}(X \cup\{x\})$, and $\operatorname{cl}(X-x)$ to mean $\operatorname{cl}(X \backslash\{x\})$.

Suppose that $B$ is an independent subset and $X, Y \subseteq B$. By monotonicity, $\operatorname{cl}(X \cap Y) \subseteq \operatorname{cl}(X)$ and $\operatorname{cl}(X \cap Y) \subseteq \operatorname{cl}(Y)$, so $\operatorname{cl}(X \cap Y) \subseteq \operatorname{cl}(X) \cap \operatorname{cl}(Y)$.

Now suppose $z \in \operatorname{cl}(X) \cap \operatorname{cl}(Y) \backslash \operatorname{cl}(X \cap Y)$. By finite character, there are $X_{0} \subseteq_{\text {fin }} X$ and $Y_{0} \subseteq_{\text {fin }} Y$ such that $z \in \operatorname{cl}\left(X_{0}\right) \cap \operatorname{cl}\left(Y_{0}\right) \backslash \operatorname{cl}(X \cap Y)$. By exchange, there is $x \in X_{0} \backslash Y$ such that $\operatorname{cl}\left(X_{0}\right)=\operatorname{cl}\left(X_{0} z-x\right)$. Similarly, there is $y \in Y_{0} \backslash X$ such that $\operatorname{cl}\left(Y_{0}\right)=\operatorname{cl}\left(Y_{0} z-y\right)$. Hence

$$
C:=\operatorname{cl}\left(X_{0} Y_{0}\right)=\operatorname{cl}\left(X_{0} Y_{0} z-x-y\right)
$$

but $X_{0} \cup Y_{0}$ is an independent set and so $\operatorname{dim} C=\left|X_{0} \cup Y_{0}\right|$, and yet $C$ is generated by a set of size $\left|X_{0} \cup Y_{0}\right|-1$, which is a contradiction. Hence no such $z$ exists, and $\operatorname{cl}(X) \cap \operatorname{cl}(Y)=\operatorname{cl}(X \cap Y)$ as required.

We translate Zilber's excellence criterion (which can be proved directly in examples) to a Shelah-style criterion.

Lemma 3.2 (Excellence - Shelah style). Let $\mathcal{C}$ be a quasiminimal excellent class, let $H, H^{\prime} \in \mathcal{C}$ and let $C \subseteq H$ be a countable crown. Then every closed partial embedding $f: H \rightarrow H^{\prime}$ with preimage $C$ extends to a closed embedding $\hat{f}: \mathrm{cl}_{H}(C) \hookrightarrow H^{\prime}$.

Proof. Let $\bar{C}=\mathrm{cl}_{H}(C)$ and let $\bar{C}^{\prime}=\mathrm{cl}_{H^{\prime}}(f(C))$. They are both countable, so choose an ordering of each of length $\omega$.

Inductively we construct partial embeddings $f_{n}: H \rightarrow H^{\prime}$ for $n \in \mathbb{N}$ such that for each $n$ :

- $\operatorname{preim}\left(f_{n}\right)$ is finite,

- $f_{n} \subseteq f_{n+1}$, and

- $f_{n} \cup f$ is a partial embedding.

Take $f_{0}=\emptyset$. We construct the $f_{n}$ for $n>0$ via the back and forth method, going forth for odd $n$ and back for even $n$.

For odd $n$, let $a$ be the least element of $\bar{C} \backslash \operatorname{preim}\left(f_{n-1}\right)$. Then the set $\operatorname{preim}\left(f_{n-1}\right) \cup\{a\}$ is a finite subset of $\bar{C}$, so by axiom III (excellence) and the finite character of the pregeometry there is a finite subset $C_{0}$ of $C$ such that the quantifier-free type of $\operatorname{preim}\left(f_{n-1}\right) \cup\{a\}$ over $C$ is determined over 
$C_{0}$ and $a \in \operatorname{cl}_{H}\left(C_{0}\right)$. Let $g_{n}=f_{n-1} \cup f \uparrow_{C_{0}}$. By induction, $f_{n-1} \cup f$ is a partial embedding, so $g_{n}$ is a partial embedding. By axiom II.2 ( $\aleph_{0}$-homogeneity), there is $b \in H^{\prime}$ such that $f_{n}:=g_{n} \cup\{(a, b)\}$ is a partial embedding. Since the type of preim $\left(f_{n}\right)$ over $C$ is determined over $C_{0}, f_{n} \cup f$ is a partial embedding, as required.

For even $n$, note that $f(C)$ is a crown in $H^{\prime}$ because $f$ is a closed partial embedding. Also note that the inverse of a partial embedding is a partial embedding. Hence we can perform the same process as for odd steps, reversing the roles of $H$ and $H^{\prime}$, to find $f_{n}$ whose image contains the least element of $\bar{C}^{\prime}$ not in the image of $f_{n-1}$.

Let $\hat{f}=\bigcup_{n \in \mathbb{N}} f_{n}$. Then $\hat{f}$ is an embedding extending $f$, defined on all of $\bar{C}$, whose image is all of $\bar{C}^{\prime}$. Hence $\hat{f}$ is a closed embedding.

We can now improve theorem 2.1 by removing the cardinality restriction.

Theorem 3.3. Let $\mathcal{C}$ be a quasiminimal excellent class, and let $H, H^{\prime} \in \mathcal{C}$. Let $G \subseteq H$ be empty or closed and let $f_{0}: G \rightarrow H^{\prime}$ be a closed embedding. Let $B$ be a basis of $H$ over $G$ and suppose $\psi_{B}: H \rightarrow H^{\prime}$ is an injective partial map with preimage $B$ and image an independent set over $\operatorname{Im} f_{0}$. Then $\psi:=f_{0} \cup \psi_{B}$ extends to a closed embedding $\hat{\psi}: H \rightarrow H^{\prime}$.

In particular, if $\operatorname{Im} \psi$ spans $H^{\prime}$ then $\hat{\psi}$ is an isomorphism.

Proof. If $\operatorname{dim} H$ is finite then we are done by theorem 2.1. so we assume $\operatorname{dim} H$ is infinite. If $G$ is empty or finite dimensional then we may extend $G$ to a closed subset of dimension $\aleph_{0}$, using theorem 2.1. We first prove the theorem assuming that $\operatorname{dim} G=\aleph_{0}$. The case where $\operatorname{dim} G>\aleph_{0}$ will be discussed afterwards.

For each finite subset $X \subseteq_{\text {fin }} B$, we will construct a closed embedding $f_{X}: \mathrm{cl}_{H}(G X) \rightarrow H^{\prime}$ such that

- whenever $Y \subseteq X$, we have $f_{Y} \subseteq f_{X}$, and

- $f_{X} \uparrow_{X}=\psi \uparrow_{X}$.

We construct the $f_{X}$ by well-founded induction on the partial order of finite subsets of $B$. Take $f_{\emptyset}:=f_{0}$.

Suppose that $X \subseteq_{\text {fin }} B, X \neq \emptyset$, and we already have $f_{Y}$ for all proper subsets $Y$ of $X$. Let $g_{X}=\bigcup_{Y \subsetneq X} f_{Y}$. Using lemma 3.1, we see that if $x \in \operatorname{preim}\left(f_{Y_{1}}\right) \cap \operatorname{preim}\left(f_{Y_{2}}\right)$ for $Y_{1}, Y_{2} \subsetneq X$ then $x \in \operatorname{preim}\left(f_{Y_{1} \cap Y_{2}}\right)$, and so by the inductive hypothesis $f_{Y_{1}}(x)=f_{Y_{2}}(x)$. Hence $g_{X}$ is a well-defined partial function. We must show that $g_{X}$ is a partial embedding.

Say $X=\left\{x_{1}, \ldots, x_{n}\right\}$, and for $i=1, \ldots, n$ let $Y_{i}=X \backslash\left\{x_{i}\right\}$. Let $C_{k}=\bigcup_{i=1}^{k} \operatorname{cl}_{H}\left(G Y_{i}\right)$ and let $h_{k}=\bigcup_{i=1}^{k} f_{Y_{i}}$. So $g_{X}=h_{n}$.

We prove by induction on $k$ that $h_{k}$ is a partial embedding. The $k=1$ case is immediate. For the induction step, take tuples $a \in C_{k-1}$ and $b \in$ 
$\mathrm{cl}_{H}\left(G Y_{k}\right)$. We construct an automorphism of a submodel of $H^{\prime}$ which has the effect of moving the parameters $a$ inside $\mathrm{cl}_{H}\left(G Y_{k}\right)$. Let $A \subseteq_{\text {fin }} G$ such that $a, b \in \mathrm{cl}_{H}(A X)$, and let $G_{0}=\operatorname{cl}_{H}(A)$. Let $z \in G \backslash G_{0}$, let $H_{0}=\mathrm{cl}_{H}\left(G_{0} X z\right)$, and let $H_{0}^{\prime}=\operatorname{cl}_{H^{\prime}}\left(\psi\left(G_{0} X z\right)\right)$. By theorem 2.1, there is an automorphism $\sigma$ of $H_{0}$, fixing $\operatorname{cl}_{H}\left(G_{0} Y_{k}\right)$ and swapping $x_{k}$ and $z$.

The idea is to compare $h_{k}$ on $H_{0}$ with the composite embedding $\tau=$ $\sigma^{\prime-1} f_{Y_{k}} \sigma$, where $\sigma^{\prime}$ is the automorphism of $H_{0}^{\prime}$ which "corresponds" to $\sigma$. To have a notion of what "corresponds" means, we must choose a suitable isomorphism between $H_{0}$ and $H_{0}^{\prime}$. Fortunately, the condition of being "suitable" is weaker than the compatibility condition we are trying to prove!

Let $e$ be a closed embedding $e: \operatorname{cl}_{H}(G X) \rightarrow H^{\prime}$ extending $h_{k-1} \cup \psi \Upsilon_{X}$. For $k=2$, such an $e$ exists because $X=Y_{1} \cup\left\{x_{1}\right\}$, so $h_{1} \cup\left\{\left(x_{1}, \psi\left(x_{1}\right)\right\}\right.$ extends to some $e$ by theorem 2.1. For $k \geqslant 3, C_{k-1}$ is a crown whose closure is $\mathrm{cl}_{H}(G X)$, so $h_{k}$ extends to some $e$ by lemma 3.2. Let $\sigma^{\prime}=e \sigma e^{-1}$, and let $\tau=\sigma^{\prime-1} f_{Y_{k}} \sigma=e \sigma^{-1} e^{-1} f_{Y_{k}} \sigma$.

Write $Y_{i k}$ for $Y_{i} \cap Y_{k}$. The tuple $a \in \bigcup_{i=1}^{k-1} \mathrm{cl}_{H}\left(G_{0} Y_{i}\right)$, so

$$
\sigma(a) \in \bigcup_{i=1}^{k-1} \mathrm{cl}_{H}\left(G_{0} Y_{i k} z\right) \subseteq \bigcup_{i=1}^{k-1} \mathrm{cl}_{H}\left(G Y_{i k}\right) \subseteq \operatorname{preim}\left(h_{k-1}\right) .
$$

By hypothesis, $f_{Y_{k}}$ agrees with $f_{Y_{i}}$ on $\mathrm{cl}_{H}\left(G Y_{i k}\right)$, hence $f_{Y_{k}}$ agrees with $h_{k-1}$ on $\bigcup_{i=1}^{k-1} \mathrm{cl}_{H}\left(G Y_{i k}\right)$. Also $e$ and $h_{k}$ both extend $h_{k-1}$, so

$$
\tau(a)=e \sigma^{-1} e^{-1} f_{Y_{k}} \sigma(a)=h_{k-1} \sigma^{-1} h_{k-1}^{-1} h_{k-1} \sigma(a)=h_{k-1}(a)=h_{k}(a) .
$$

The tuple $b \in \operatorname{cl}_{H}\left(G_{0} Y_{k}\right)$, so it is fixed by $\sigma$. The embeddings $f_{Y_{k}}$ and $e$ preserve the closure, so $e^{-1} f_{Y_{k}}(b)$ is fixed by $\sigma^{-1}$. So

$$
\tau(b)=e \sigma^{-1} e^{-1} f_{Y_{k}} \sigma(b)=e e^{-1} f_{Y_{k}}(b)=f_{Y_{k}}(b)=h_{k}(b) .
$$

Thus for any quantifier-free formula $R$,

$$
H \models R(a, b) \Longleftrightarrow H^{\prime} \models R(\tau(a), \tau(b)) \Longleftrightarrow H^{\prime} \models R\left(h_{k}(a), h_{k}(b)\right) .
$$

This holds for any tuples $a, b$ (for a suitable choice of $\tau$ ) and so $h_{k}$ is a partial embedding. In particular, $g_{X}=h_{n}$ is a partial embedding. It is a union of finitely many closed partial embeddings, hence is a closed partial embedding.

By lemma 3.2, $g_{X}$ extends to a closed embedding $f_{X}: \operatorname{cl}_{H}(G X) \rightarrow H^{\prime}$. Thus, by induction, we have compatible embeddings $f_{X}$ for every $X \subseteq_{\text {fin }}$ $B$. Let $\hat{\psi}=\bigcup_{X \subseteq_{\text {fin } B}} f_{X}$, a closed embedding. By the finite character of the closure, $H=\bigcup_{X \subseteq_{\text {fin } B}} \operatorname{cl}_{H}(G X)$. Hence $\hat{\psi}$ is a total map on $H$. That completes the proof in the case where $\operatorname{dim} G \leqslant \aleph_{0}$. 
If $\operatorname{dim} G>\aleph_{0}$, let $G^{\prime}$ be a closed subset of $G$ of dimension $\aleph_{0}$, and let $B_{1}$ be a basis of $G$ over $G^{\prime}$. Inductively construct embeddings $f_{X}$ as before, with $G^{\prime}$ in place of $G$ and $B \cup B_{1}$ in place of $B$, except that for $X \subseteq_{\text {fin }} B_{1}$ take $f_{X}=f_{0} \uparrow_{\mathrm{cl}_{H}\left(G^{\prime} X\right)}$. Then the map $\hat{\psi}$ obtained will extend $\psi$ as required.

Corollary 3.4 (Uniqueness of models). Models of a quasiminimal excellent class are determined up to isomorphism by their dimension. There is at most one model of any uncountable cardinality.

A small modification of the proof of theorem 3.3 , similar to the case of proposition 2.3, gives the following full homogeneity and quantifier elimination statements.

Proposition 3.5. Let $\mathcal{C}$ be a quasiminimal excellent class.

- Any model in $\mathcal{C}$ is $\aleph_{0}$-homogeneous over the empty set and over every closed submodel.

- The Galois types of finite tuples over the empty set and over closed submodels are equal to the quantifier-free L-types.

\section{Existence of models}

The axioms for a quasiminimal excellent class do not imply that any models exist at all. If there is a model of dimension $\kappa$, then for any cardinal $\lambda<\kappa$ there is a model of dimension $\lambda$, by axiom I.2. Hence the models of a quasiminimal excellent class are indexed by some initial segment of the class of cardinals. There is nothing in the axioms to say that we cannot have a proper initial segment - indeed any initial segment of any quasiminimal excellent class is also a quasiminimal excellent class. There are also quasiminimal excellent classes with only models of finite dimension. To exclude these degenerate cases, we consider additional axioms. We will see that they exclude only these degenerate cases.

Definition 4.1. For an ordinal $\lambda$, a chain of $L$-structures of length $\lambda$ consists of $L$-structures $\left(M_{\mu}\right)_{\mu<\lambda}$ and for each $\mu \leqslant \nu<\lambda$, an embedding $f_{\mu, \nu}: M_{\mu} \rightarrow$ $M_{\nu}$ such that if $\mu \leqslant \nu \leqslant \rho<\lambda$ then $f_{\nu, \rho} \circ f_{\mu, \nu}=f_{\mu, \rho}$, and $f_{\mu, \mu}$ is the identity on $M_{\mu}$. Equivalently, it is a functor from the ordinal $\lambda$ considered as an ordered set (and hence as a category) into the category of $L$-structures and $L$-embeddings. By the union of the chain we mean its colimit. In the case when the $f_{\mu, \nu}$ are inclusion maps of substructures, this union is the actual union of the underlying sets.

\section{IV: Chains}


IV.1 The category $\mathcal{C}$ has unions of chains of all ordinal lengths. That is, suppose $\left(H_{\mu}\right)_{\mu<\lambda}$ is a chain of models of $\mathcal{C}$ with closed embeddings. Let $H$ be the union of the chain as an $L$-structure, and for $X \subseteq H$, define $c l_{H}(X)=\bigcup_{\mu<\lambda} \operatorname{cl}_{H_{\mu}}\left(X \cap H_{\mu}\right)$. Then $\left\langle H, \mathrm{cl}_{H}\right\rangle \in \mathcal{C}$.

IV.2 $\mathcal{C}$ contains an infinite dimensional model.

It is easy to show that a quasiminimal excellent class which satisfies axiom IV.1 (unions of chains) is an abstract elementary class with Löwenheim cardinal at most $\aleph_{0}$. We have no further use for abstract elementary classes in this paper, so refer the reader to [Bal07] for the definition and the ideas around it.

By the countable closure property, the cardinality of an infinite-dimensional model of a quasiminimal excellent class is equal to its dimension. Thus there is at most one model of each uncountable cardinality. We say that a class is uncountably categorical iff there is exactly one model of each uncountable cardinality.

Theorem 4.2 (Existence of models). Let $\mathcal{C}$ be a quasiminimal excellent class satisfying axiom $I V$. Then for every cardinal $\kappa$ there is a unique $H \in \mathcal{C}$ of dimension $\kappa$. In particular, $\mathcal{C}$ is uncountably categorical. Conversely, any uncountably categorical quasiminimal excellent class satisfies axiom IV.

Proof. We have already proved the uniqueness of models of each cardinal dimension, and it remains to prove their existence. By induction on ordinals $\lambda$ we construct the initial segment of length $\lambda$ of a chain $\left(M_{\mu}\right)_{\mu \in \mathrm{ON}}$ in $\mathcal{C}$ where each $M_{\mu}$ has a chosen basis indexed by $\mu$, and the inclusion maps $M_{\mu} \hookrightarrow M_{\nu}$ of the chain extend the inclusion maps $\mu \hookrightarrow \nu$ of the ordinals (identified with the chosen bases). Here $\mathrm{ON}$ is the ordered class of ordinals.

By axiom IV.2, there is $M \in \mathcal{C}$ with $\operatorname{dim} M=\kappa$, for some infinite cardinal $\kappa$. Choose an ordering of a basis of $M$ of length the initial ordinal $\alpha$ of size $\kappa$. Taking the closures of the initial segments of this basis gives the chain $\left(M_{\mu}\right)_{\mu<\alpha}$ in $\mathcal{C}$.

There are two cases for the inductive step. If $\lambda$ is a successor ordinal $\lambda=\nu+1$ then the model $M_{\nu}$ has dimension $|\lambda|$, so we take $M_{\lambda}=M_{\nu}$. We choose a new ordering of length $\lambda$ for the basis, and, using theorem 3.3 , we choose a closed embedding $M_{\nu} \hookrightarrow M_{\lambda}$ extending the inclusion of bases.

If $\lambda$ is a limit ordinal then by induction we have a chain $\left(M_{\mu}\right)_{\mu<\lambda}$ in $\mathcal{C}$. By axiom IV.1, the union of this chain lies in $\mathcal{C}$, and the inclusion maps of the $M_{\mu}$ into the limit are closed. It has a basis indexed by $\lambda$, so we take it as $M_{\lambda}$. That proves one direction of the theorem.

For the converse, suppose $\mathcal{C}$ is uncountably categorical, and $\left(H_{\nu}\right)_{\nu<\lambda}$ is a chain in $\mathcal{C}$. Let $\kappa$ be a cardinal which is an upper bound for the dimensions 
of the $H_{\nu}$, and for $|\lambda|$, and let $\alpha$ be the initial ordinal of $\kappa$. Then the model $M_{\kappa}$ in $\mathcal{C}$ of dimension $\kappa$ can be written as the union of a chain $\left(M_{\mu}\right)_{\mu<\alpha}$, as above. The chain $\left(H_{\nu}\right)_{\nu<\lambda}$ is isomorphic to a subchain of $\left(M_{\mu}\right)_{\mu<\alpha}$ (possibly with repeats), so its union is naturally a closed subset of $M_{\kappa}$. Hence the union lies in $\mathcal{C}$. Thus $\mathcal{C}$ satisfies IV.1. Axiom IV.2 is immediate.

\section{Definability}

This section is mainly about the definability of a quasiminimal excellent class, but we first give a result about definable sets within a class. The motivation for the word quasiminimal is that every definable set is countable or cocountable.

Lemma 5.1 (Quasiminimality). Let $\mathcal{C}$ be a quasiminimal excellent class, and let $H \in \mathcal{C}$.

- If $X \subseteq H$ and $a, b \in H \backslash \mathrm{cl}_{H}(X)$ then there is $\sigma \in \operatorname{Aut}(H / X)$ such that $\sigma(a)=b$.

- Every subset of $H$ which is definable with countably many parameters in any logic respecting L-automorphisms (for example $L_{\infty, \omega}$ or $\left.L_{\omega_{1}, \omega}(Q)\right)$ is either countable or cocountable.

Proof. The first part is immediate from theorem 3.3 . For the second part, let $S \subseteq H$ be definable from a countable parameter set $A$. Then $H \backslash \mathrm{cl}_{H}(A)$ is a single orbit of $\operatorname{Aut}(H / A)$, and is cocountable, and either $S$ does not meet $H \backslash \operatorname{cl}_{H}(A)$ or $S$ contains $H \backslash \operatorname{cl}_{H}(A)$.

There is no assumption that the language $L$ should be countable, but in fact we may assume that it is.

Proposition 5.2. Let $\mathcal{C}$ be a quasiminimal excellent class, in a language $L$. Then there is a countable sublanguage $L^{0}$ of $L$ such that the class $\mathcal{C}^{0}$ of reducts to $L^{0}$ of models in $\mathcal{C}$ is also a quasiminimal excellent class, and the reduct map $\mathcal{C} \rightarrow \mathcal{C}^{0}$ is an isomorphism of categories.

Proof. Let $M$ be a countable model, $G$ a closed submodel of $M$ or $\emptyset$, and $a, b$ be $n$-tuples from $M$, and suppose the quantifier-free types $\operatorname{qftp}(a / G)$ and $\operatorname{qftp}(b / G)$ are different. Then there is a symbol from the signature of $L$ which witnesses this. Up to isomorphism, there are only countably many such tuples $\langle M, G, a, b\rangle$. Hence there is a countable sublanguage $L^{0}$ of $L$ which witnesses all such differences of quantifier-free types. By induction using $\aleph_{0^{-}}$ homogeneity over models, partial $L^{0}$-embeddings and partial $L$-embeddings of countable models coincide. 
The language $L$ is finitary (that is, every symbol has finite arity), so a map between two models is an $L$-embedding precisely when its restriction to every finitely generated substructure is an $L$-embedding. By the countable closure property, an embedding is closed precisely when its restriction to every countable submodel is closed. Hence partial (and total) closed $L^{0}$ embeddings and closed $L$-embeddings of any models coincide. In particular, quantifier-free $L^{0}$-types coincide with quantifier-free $L$-types. Hence for each relation symbol $R$ of $L$, there is an quantifier-free $L^{0}$-formula $\theta_{R}(\bar{x})$ such that for any $M \in \mathcal{C}, M \models(\forall \bar{x})\left[R(\bar{x}) \leftrightarrow \theta_{R}(\bar{x})\right]$. This gives a unique way of expanding a model in $\mathcal{C}^{0}$ to a model in $\mathcal{C}$, which in turn gives an inverse to the reduct map.

Lemma 5.3 ( $L_{\omega_{1}, \omega}$-definability of the pregeometry). Let $\mathcal{C}$ be a quasiminimal excellent class. For each $n \in \mathbb{N}$ there is a quantifier-free $L_{\omega_{1}, \omega}$-formula $\pi_{n}\left(x, y_{1}, \ldots, y_{n}\right)$ such that for each $H \in \mathcal{C}$ and each $a, b_{1}, \ldots, b_{n} \in H$, we have

$$
a \in \mathrm{cl}_{H}(\bar{b}) \text { iff } H \models \pi_{n}(a, \bar{b}) .
$$

Proof. By proposition 5.2 we may assume the language is countable. For each $n \in \mathbb{N}$, let $M_{n}$ be the model of dimension $n$ (if it exists, and the model of maximum dimension if it does not). For each $n$, every quantifier-free $n$ type is realised in the closure of its realization, hence in $M_{n}$, hence there are only countably many quantifier-free $n$-types over $\emptyset$. The types are given by quantifier-free $L_{\omega_{1}, \omega}$-formulas, say $\left(\beta_{j}(\bar{y})\right)_{j \in \mathbb{N}}$. By the same argument, for each $j$ and $\bar{b}$ of type $\beta_{j}$, there are only countably many 1 -types over $\bar{b}$ of elements in the closure of $\bar{b}$, and their types are also given by quantifier-free $L_{\omega_{1}, \omega}$-formulas, say $\left(\gamma_{i j}(x, \bar{b})\right)_{i \in \mathbb{N}}$. So the formula

$$
\pi_{n}(x, \bar{y}) \equiv \bigvee_{j \in \mathbb{N}}\left(\beta_{j}(\bar{y}) \wedge \bigvee_{i \in \mathbb{N}} \gamma_{i j}(x, \bar{y})\right)
$$

works for $M_{n+1}$. If $H \in \mathcal{C}$ and $a, b_{1}, \ldots, b_{n} \in H$, then either there is a closed embedding $H \hookrightarrow M_{n+1}$, or there is a closed embedding $M_{n+1} \hookrightarrow H$ whose image contains $a, b_{1}, \ldots, b_{n}$. In each case, $H \models \pi_{n}(a, \bar{b})$ iff $M_{n+1} \models \pi_{n}(a, \bar{b})$ since $L$-embeddings preserve quantifier-free $L_{\omega_{1}, \omega}$-formulas, and $a \in \operatorname{cl}_{H}(\bar{b})$ iff $a \in \mathrm{cl}_{M_{n+1}}(\bar{b})$ by lemma 1.4 . Hence the same formulas $\pi_{n}$ work for every $H \in \mathcal{C}$.

Lemma 5.4. Let $\mathcal{C}$ be a quasiminimal excellent class, let $f: M \rightarrow N$ be a closed embedding in $\mathcal{C}$ and suppose that $\operatorname{dim} M \geqslant \aleph_{0}$. Then $f$ is an $L_{\omega_{1}, \omega^{-}}$ embedding. 
Proof. We prove by induction on formulas that for any $L_{\omega_{1}, \omega}$ formula $\theta(\bar{x})$ and any $\bar{a} \in M$, we have $M \models \theta(\bar{a}) \Longleftrightarrow N \models \theta(\bar{a})$.

$f$ is an $L$-embedding, so atomic formulas are preserved.

The cases $\theta(\bar{x}) \equiv \bigwedge_{i \in I} \theta_{i}(\bar{x})$ and $\theta(\bar{x}) \equiv \neg \theta_{0}(\bar{x})$ are immediate.

If $\theta(\bar{x}) \equiv \exists y \varphi(y, \bar{x})$ then the left to right case is immediate. Suppose $N \models \exists y \varphi(y, \bar{a})$. Then for some $b \in N, N \models \varphi(b, \bar{a})$. If $b \in M$ then we are done. Suppose $b \notin M$. Since $\operatorname{cl}(\bar{a})$ is finite dimensional, we can choose $c \in M \backslash \operatorname{cl}(\bar{a})$. The point $b$ is independent from $M$, so by lemma 5.1 there is an automorphism of $N$ fixing $\operatorname{cl}(\bar{a})$ and swapping $b$ and $c$. So $N \models \varphi(c, \bar{a})$ and, by the inductive hypothesis, $M \models \varphi(c, \bar{a})$. Hence $M \models \exists y \varphi(y, \bar{a})$.

Hence $f$ is an $L_{\omega_{1}, \omega}$-embedding as required.

It is easy to extend the proof to show that if $\operatorname{dim} M \geqslant \aleph_{1}$ then $f$ is an $L_{\omega_{1}, \omega}(Q)$-embedding.

Theorem 5.5. Let $\mathcal{C}$ be a quasiminimal excellent class in a countable language, with an infinite dimensional model. For each $n \leqslant \omega$, let $M_{n}$ be the model of dimension $n$, and let $\sigma_{n}$ be its Scott sentence. Let

$$
\Sigma=\left[\bigvee_{n \leqslant \omega} \sigma_{n}\right] \wedge\left[\bigwedge_{n \in \mathbb{N}}\left(\forall y_{1} \ldots, y_{n}\right) \neg(Q x) \pi_{n}\left(x, y_{1}, \ldots, y_{n}\right)\right]
$$

where $Q$ is the quantifier "there exist uncountably many". Then $\operatorname{Mod}(\Sigma)$ is an uncountably categorical quasiminimal excellent class containing $\mathcal{C}$. Furthermore, if $\mathcal{C}$ satisfies axiom $I V$ then $\mathcal{C}=\operatorname{Mod}(\Sigma)$.

Proof. We check axioms I-IV for $\operatorname{Mod}(\Sigma)$. The statement that the $\pi_{n}$ define a pregeometry can be axiomatized as an $L_{\omega_{1}, \omega}$-sentence, and it is true in each $M_{n}$, hence it follows from each $\sigma_{n}$. The countable closure property is explicit in $\Sigma$, hence axiom I.1 holds. Axiom I.3 holds because the pregeometry is defined by the quantifier-free $L_{\omega_{1}, \omega}$ formulas $\pi_{n}$. From these axioms we get the notion of dimension for each model of $\Sigma$, and we also get the notion of a closed embedding.

For each uncountable cardinal $\kappa$, let $\mathcal{D}_{\kappa}=\{N \models \Sigma \mid \operatorname{dim} N<\kappa\}$. Axioms I.1 and I.3 hold for each $\mathcal{D}_{\kappa}$ as well.

Axioms II and III are statements about the countable models. Any countable model of $\Sigma$ must be one of the $M_{n}$ for $n \leqslant \omega$, since Scott sentences are $\aleph_{0}$-categorical. Thus the countable models of $\Sigma$ are just the countable models of $\mathcal{C}$. Hence $\operatorname{Mod}(\Sigma)$ and each $\mathcal{D}_{\kappa}$ satisfy II and III. The infinite-dimensional model $M_{\omega}$ lies in $\operatorname{Mod}(\Sigma)$ and each $\mathcal{D}_{\kappa}$, so IV.2 holds. It remains to prove axioms I.2 and IV.1. We prove two related families of statements: 
$\left.1_{\kappa}\right) \mathcal{D}_{\kappa}$ satisfies I.2 and hence is a quasiminimal excellent class.

$2_{\kappa}$ ) If $H \models \Sigma$ and $N$ is a closed subset of $H$ with $\operatorname{dim} N=\kappa$ then $N \models \Sigma$.

We must prove statement 2 for all $\kappa$, and statement 1 for uncountable $\kappa$. First we prove statement 2 for countable $\kappa$. For $n \in \mathbb{N}$, let $x_{1}, \ldots, x_{n}$ be variables not occuring in $\sigma_{n}$, and let $\sigma_{n}^{\prime}\left(x_{1}, \ldots, x_{n}\right)$ be the $L_{\omega_{1}, \omega}$-formula obtained from $\sigma_{n}$ by recursively replacing all quantified subformulas of the form $\exists y[\varphi(y)]$ by $\exists y\left[\pi_{n}\left(y, x_{1} \ldots, x_{n}\right) \wedge \varphi(y)\right]$, for any subformula $\varphi(y)$ (and similarly for universal quantifiers). Let $\operatorname{Indep}_{n}\left(x_{1}, \ldots, x_{n}\right)$ be the formula $\bigwedge_{i=1}^{n} \neg \pi_{i-1}\left(x_{i}, x_{1}, \ldots, x_{i-1}\right)$. Then $\operatorname{Indep}_{n}\left(x_{1}, \ldots, x_{n}\right)$ says that the $x_{i}$ are clindependent, and $\sigma_{n}^{\prime}\left(x_{1}, \ldots, x_{n}\right)$ says that the closure of the $x_{i}$ is a model of $\sigma_{n}$. Thus for each $m \leqslant \omega$, and each $n \in \mathbb{N}$,

$$
\sigma_{m} \vdash\left(\forall x_{1}, \ldots, x_{n}\right)\left[\operatorname{Indep}_{n}\left(x_{1}, \ldots, x_{n}\right) \rightarrow \sigma_{n}^{\prime}\left(x_{1}, \ldots, x_{n}\right)\right] .
$$

Hence if $N$ is finite dimensional, it is a model of $\Sigma$.

Now suppose $\operatorname{dim} N=\aleph_{0}$. Let $\left(b_{n}\right)_{n<\omega}$ be a basis for $N$, and let $N_{m}=$ $\operatorname{cl}_{H}\left(\left\{b_{n} \mid n<m\right\}\right)$ for each $m<\omega$. Then $N$ is the union of the chain $\left(N_{m}\right)_{m<\omega}$ and, by the above, $N_{m} \cong M_{m}$. The union of the chain $\left(M_{m}\right)_{m \leqslant \omega}$ is $M_{\omega}$, hence $N \cong M_{\omega}$. In particular, $N \models \Sigma$. Thus $2_{\kappa}$ holds for all countable $\kappa$.

Now we prove statements $1_{\kappa}$ and $2_{\kappa}$ together for uncountable $\kappa$, by induction. Suppose inductively that $2_{\lambda}$ holds for every $\lambda<\kappa$. Then if $H \in \mathcal{D}_{\kappa}$, every closed subset of $H$ has dimension less than $\kappa$, so is a model of $\Sigma$. Hence $1_{\kappa}$ holds.

Now suppose $H \models \Sigma$ and $N$ is a closed subset of $H$ with $\operatorname{dim} N=\kappa$. Identifying $\kappa$ with its initial ordinal, let $\left(b_{\lambda}\right)_{\lambda<\kappa}$ be a basis of $N$ and let $N_{\mu}=\mathrm{cl}_{H}\left(\left\{b_{\lambda} \mid \lambda<\mu\right\}\right)$. Then $N$ is the union of the chain $\left(N_{\mu}\right)_{\omega \leqslant \mu<\kappa}$, and by induction each $N_{\mu}$ models $\Sigma$. The chain lies in $\mathcal{D}_{\kappa}$, which by the same induction hypothesis satisfies I.2 and hence is a quasiminimal excellent class. Thus by lemma 5.4, the chain is an $L_{\omega_{1}, \omega}$-chain. Each $N_{\mu}$ in the chain is infinite dimensional, thus models $\sigma_{\omega}$, and hence $N \models \sigma_{\omega}$. Also $N \subseteq H$, and $H$ has the CCP, so $N$ also has the CCP. Thus $N \models \Sigma$, that is, $2_{\kappa}$ holds. Thus, by induction, $1_{\kappa}$ and $2_{\kappa}$ hold for all uncountable $\kappa$.

Thus $\operatorname{Mod}(\Sigma)$ satisfies axiom I.2, and so is a quasiminimal excellent class. If $\left(H_{\lambda}\right)_{\lambda<\kappa}$ is any chain in $\operatorname{Mod}(\Sigma)$ then either its union is finite dimensional (and lies in $\operatorname{Mod}(\Sigma)$ ) or the chain is eventually infinite dimensional and by lemma 5.4 is eventually an $L_{\omega_{1}, \omega}$-chain. In the latter case, as above, the union $H$ of the chain is a model of $\sigma_{\omega}$. If $X$ is a finite subset of $H$ then $X \subseteq H_{\lambda}$ for some $\lambda<\kappa$, and $\operatorname{cl}_{H}(X)=\operatorname{cl}_{H_{\lambda}}(X)$. Since $H_{\lambda}$ has the CCP, this closure is countable. Hence $H$ has the $\mathrm{CCP}$, and so $H \models \Sigma$. $\operatorname{Thus} \operatorname{Mod}(\Sigma)$ 
satisfies axiom IV.1. By theorem 4.2, $\operatorname{Mod}(\Sigma)$ is an uncountably categorical quasiminimal excellent class.

If $H \in \mathcal{C}$ then either $H$ is finite dimensional in which case $H$ is $M_{n}$ for some $n \in \mathbb{N}$, or there is a closed embedding $M_{\omega} \hookrightarrow H$. By lemma 5.4, this embedding is an $L_{\omega_{1}, \omega}$-embedding, so $H \models \sigma_{\omega}$. In either case, $H \models \Sigma$. So $\mathcal{C} \subseteq \operatorname{Mod}(\Sigma)$. Since $\mathcal{C}$ satisfies I.2, it is an initial segment of $\operatorname{Mod}(\Sigma)$. That is, either $\mathcal{C}=\mathcal{D}_{\kappa}$ for some $\kappa$ or $\mathcal{C}$ satisfies IV.1 and $\mathcal{C}=\operatorname{Mod}(\Sigma)$.

Corollary 5.6. Let $\mathcal{C}$ be a quasiminimal excellent class with a model of dimension $\aleph_{0}$. Then there is a unique quasiminimal excellent class $\mathcal{C}^{\prime}$, containing $\mathcal{C}$, which is uncountably categorical.

Proof. By proposition 5.2 we may assume the language is countable. Then theorem 5.5 gives $\operatorname{Mod}(\Sigma)$ as one such $\mathcal{C}^{\prime}$. If $\mathcal{C}^{\prime \prime}$ is any uncountably categorical quasiminimal excellent class containing $\mathcal{C}$ then by theorem 4.2 it satisfies axiom IV, so by theorem 5.5 again it is equal to $\operatorname{Mod}(\Sigma)$. Hence $\mathcal{C}^{\prime}$ is unique.

From this result we see that nothing would be lost by adding axiom IV to the definition of a quasiminimal excellent class, unless perhaps there can be interesting behaviour of finite dimensional models. Some related questions are given at the end of this paper.

In theorem 5.5, the sentence $\Sigma$ depends only on the model $M_{\omega}$, because the $M_{n}$ are substructures of $M_{\omega}$. We can extract the properties of $M_{\omega}$ which are needed to produce a quasiminimal excellent class.

Corollary 5.7. Let $M$ be a countable L-structure, equipped with a pregeometry $\mathrm{cl}$, satisfying the following axioms.

\section{$I^{\prime}$ (Pregeometry)}

The pregeometry is quantifier-free $L_{\omega_{1}, \omega}$-definable, and $\operatorname{dim} M=\aleph_{0}$.

\section{II $^{\prime}\left(\aleph_{0}\right.$-homogeneity over closed sets)}

Let $G \subseteq M$ be closed or empty.

II.1' If $x, x^{\prime} \in M$ are each independent from $G$, then $\operatorname{qftp}(x / G)=$ $\operatorname{qftp}\left(x^{\prime} / G\right)$.

II. 2 Let $\bar{x}, \bar{x}^{\prime}$ be finite tuples from $M$ such that $\operatorname{qftp}(\bar{x} / G)=\operatorname{qftp}\left(\bar{x}^{\prime} / G\right)$, and let $y \in M$. Then there is $y^{\prime} \in M$ such that $\operatorname{qftp}(\bar{x} y / G)=$ $\operatorname{qftp}\left(\bar{x}^{\prime} y^{\prime} / G\right)$. 


\section{III' (Excellence)}

If $C$ is a crown in $M$ and $\bar{x}$ is a finite tuple from $\operatorname{cl}(C)$, then there is a finite subset $C_{0}$ of $C$ such that for any tuple $\bar{x}^{\prime}$,

$$
\operatorname{qftp}\left(\bar{x} / C_{0}\right)=\operatorname{qftp}\left(\bar{x}^{\prime} / C_{0}\right) \Longrightarrow \operatorname{qftp}(\bar{x} / C)=\operatorname{qftp}\left(\bar{x}^{\prime} / C\right) .
$$

Then there is a unique quasiminimal excellent class $\mathcal{C}$ which satisfies axiom $I V$ such that $\langle M, \mathrm{cl}\rangle \in \mathcal{C}$.

Proof. Let $\mathcal{C}_{0}$ be the class of $L$-structures isomorphic to a cl-closed substructure of $M$, equipped with the restriction of the pregometry cl. The axioms $\mathrm{I}^{\prime}$ - III' ensure that $\mathcal{C}_{0}$ is a quasiminimal excellent class, and $M$ itself is a model of dimension $\aleph_{0}$, so corollary 5.6 gives the unique class $\mathcal{C}$.

\section{Questions}

We conclude with some further questions.

1. Is there a class which satisfies axioms 0 , I and II, but not III? It seems likely that there is, but I do not believe that any examples are currently known.

2. Does axiom III follow from lemma 3.2] The former is Zilber's definition of excellence and the latter should be Shelah's with respect to this abstract elementary class, albeit with prime model in place of primary model.

3. If $\mathcal{C}$ is a quasiminimal excellent class with models of arbitrarily large finite dimension (and hence of all finite dimensions), there is a welldefined $L$-structure $M$ constructed as the union of all of the finite dimensional models. Is $\mathcal{C} \cup\{M\}$ necessarily a quasiminimal excellent class?

4. A similar question: can axioms II and III be weakened further so they only cover the cases where $G$ and $C$ respectively are finite-dimensional, rather than countable?

5. What quasiminimal excellent classes are there with models only of dimension up to some finite $n$, with $n \geqslant 1$, which cannot be extended to larger quasiminimal excellent classes? 


\section{References}

[Bal07] John BALdwin. Categoricity, 2007. Forthcoming monograph, URL http://www.math.uic.edu/ jbaldwin.

[Mar02] David Marker. Model theory: An Introduction, volume 217 of Graduate Texts in Mathematics. Springer-Verlag, New York, 2002.

[Zil05] Boris ZILBER. A categoricity theorem for quasi-minimal excellent classes. In Logic and its applications, volume 380 of Contemp. Math., pp. 297-306. Amer. Math. Soc., Providence, RI, 2005. 